\title{
Modelling the evolution of uncertainty levels during design
}

\author{
David C. Wynn · Khadidja Grebici · P. John Clarkson
}

Cite this article as:

Wynn, DC, Grebici, K and Clarkson, PJ (2011) "Modelling the evolution of uncertainty levels during design" International Journal on Interactive Design and Manufacturing, Vol. 5, No. 3, pp. 187-202. DOI 10.1007/s12008-011-0131-y

The final publication is available at www.springerlink.com

\begin{abstract}
Design work involves uncertainty that arises from, and influences, the progressive development of solutions. This paper analyses the influences of evolving uncertainty levels on the design process. We focus on uncertainties associated with choosing the values of design parameters, and do not consider in detail the issues that arise when parameters must first be identified. Aspects of uncertainty and its evolution are discussed, and a new task-based model is introduced to describe process behaviour in terms of changing uncertainty levels. The model is applied to study two process configuration problems based on aircraft wing design: one using an analytical solution and one using MonteCarlo simulation. The applications show that modelling uncertainty levels during design can help assess management policies, such as how many concepts should be considered during design and to what level of accuracy.
\end{abstract}

Keywords Uncertainty levels - Design process model · Discrete-event simulation

\section{Introduction}

The design process can be viewed as a system of interrelated activities that are performed to increase knowledge — or to reduce uncertainty — about the design solution. This paper explores how design processes are influenced by changing levels of uncertainty in the emerging design. We focus on situations where the solution principles are well-established and the architectures of the product and its design process vary only incrementally from previous projects. In these cases the key design parameters and models are usually known from the outset, and the processes by which their values are determined can often be described. Prior to a more detailed analysis in forthcoming sections, we use the term 'uncertainty' in a high-level sense to encompass many different issues, including: lack of definition, lack of knowledge and lack of trust in knowledge.
We draw on an example of aircraft wing design to argue that design processes can be enhanced by better understanding the evolution of uncertainty levels associated with the emerging design. A task-based simulation model is introduced based on the assumptions that (1) reducing uncertainty about the design is an objective of the design process; and (2) levels of uncertainty influence how the process unfolds. We discuss how tasks in the design process can be influenced by the uncertainty levels associated with their inputs, and explain how these levels and influences can be incorporated in a design process simulation. Two illustrative applications show how the modelling approach can help understand the impact of uncertainty levels on a design process, and show how the uncertainty-oriented simulation can be used to assess potential process improvements.

\section{Case study: aircraft wing design}

The arguments throughout this paper are illustrated using a case study: the design of an aircraft wing. Wing design provides a good example of a complex engineering process, in which multi-disciplinary teams resolve design problems where the main parameters (but not their values) are well-defined from the beginning. We draw on interviews with stress engineers and designers working on structural analysis in a major aircraft manufacturer [1]. However, most features of wing design described here are common across the industry as discussed by other authors (e.g., [2]).

\subsection{Overview of the design problem}

Wing design requires many parameters to be determined, ranging from high-level configuration to low-level parameters such as the thickness of the skin. Choices made while designing other aircraft sub-systems, such as the fuselage, landing gear and engines, impact on the wing design and vice versa. Interface management is thus 
an important challenge during design. Changes in interface parameters, or in requirements, can occur at any time and necessitate rework.

One of the main challenges faced by the engineers interviewed during the study is to design a structure that can resist loads without buckling. In overview, two main types of load must be considered. Surface forces or external loads act upon the skin, and body forces or internal loads act over the volume of the structure. Loads derive from sources including the wing weight and the pressure distribution over the surface. They result in direct loads, bending, shear and torsion in all parts of the structure. Designers must consider not only of the static structure of parts and their interfaces, but also the dynamic behaviour of the wing. For instance, stresses on the leading edge must be considered under the combined effect of pressure loads and movement loads.

Most design decisions must consider multiple operating scenarios, such as steady flight, manoeuvre and gust conditions. Many other requirements and constraints must also be considered. These include weight and certification requirements, as well as design constraints such as material properties and production tolerances.

\subsection{Design decomposition}

Some of the main components of a wing are depicted in Fig. 1. Their structural functions include:

- Skin Resists the distributed aerodynamic pressure loads and distributes concentrated loads, such as those arising from the undercarriage, into the structure. The skin also redistributes stress around discontinuities such as the undercarriage wells.

- Ribs Determine the cross-section of the aerofoil. The design of each rib is governed by its span-wise position in the wing and by the loads it must support. Ribs close to the wing root absorb and transmit large concentrated loads, including those from the undercarriage and fuselage.

- Stringers Divide the skin into small panels, increasing the buckling and failure stresses without requiring the skin thickness and therefore weight to be increased.

- Spars Comprised from spar webs and flanges, spars provide much of the wing's structural integrity.

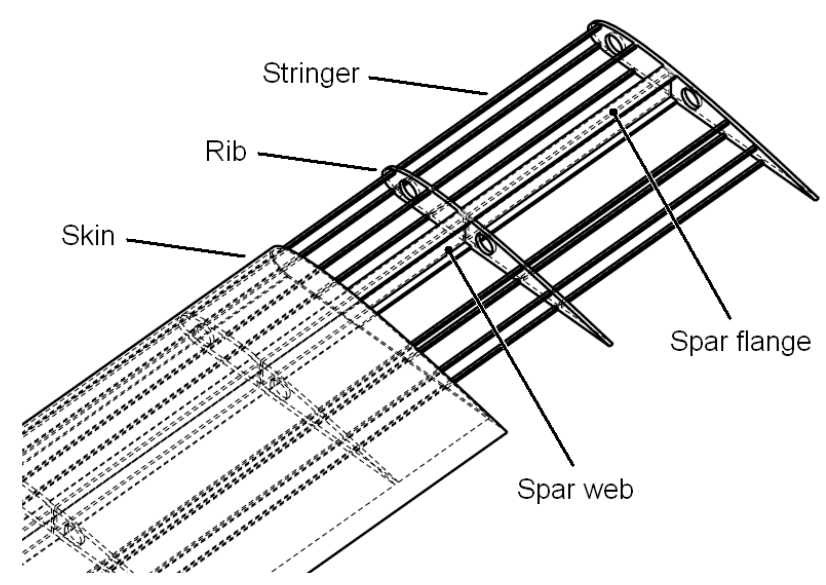

Fig. 1. Some examples of parts in an aircraft wing

\subsection{Design descriptions: data and models}

During design, information about the wing, its parts and performance is created and iteratively refined. This information is not accessed and modified directly, but is expressed through mediating descriptions suitable for different design and analysis activities. For instance, a part might be described concurrently in the form of CAD models, CFD models and FE models. These descriptions of the design are supplemented by the knowledge of process participants, much of which is not documented or made explicit. The different types of design information used during the process describe the design in an overlapping way and must be considered simultaneously. This relationship between the design, its descriptions and the design process is a source of significant complexity in many design processes [3].

\subsection{Design process}

The wing design process, as might occur in practice, begins with definition of main parameters such as length, sweep and chord. Aerodynamics specialists use these basic parameters to define wing surface geometry. Designers then define the physical parts, and the engine size must be determined. The wing and overall system design is analysed with respect to different manoeuvres, mission profiles, etc. If requirements are not met, changes to the design definition are made and analysis tasks revisited. Examples of specific tasks performed during the design process are:

- Modelling, updating and meshing models of the parts.

- Assembling FE models of the wing from part models.

- Calculating and analysing load distributions.

- Calculating the aerodynamic pressure distribution over the wing surface.

The wing parts are incrementally refined through an iterative process of definition, analysis and evaluation. Engineers in different teams and from different disciplines consider multiple descriptions and requirements. Many passes of the process may be required. On each cycle, the designers create information that may inform subsequent decisions they make.

Figure 2 draws on the case study and literature on wing design to summarise part of a generic process as it might be carried out in practice. The figure suggests a fixed sequence of activities that are repeated the same way on each iteration. This is a sanitised view that arises from the level of abstraction and structure chosen for the diagram. In reality, designers react to emerging issues when determining the focus for their attention on each cycle. Not all tasks need be performed each time; for instance, engines would not be re-sized on every iteration. The duration of some tasks is determined by how much effort designers choose to expend at that point in the design process. This can be influenced by the levels of uncertainty associated with the task's input information. For instance, there would be little benefit in carefully refining wing geometry prior to stress analysis if the requirements for the geometry were expected to change. 


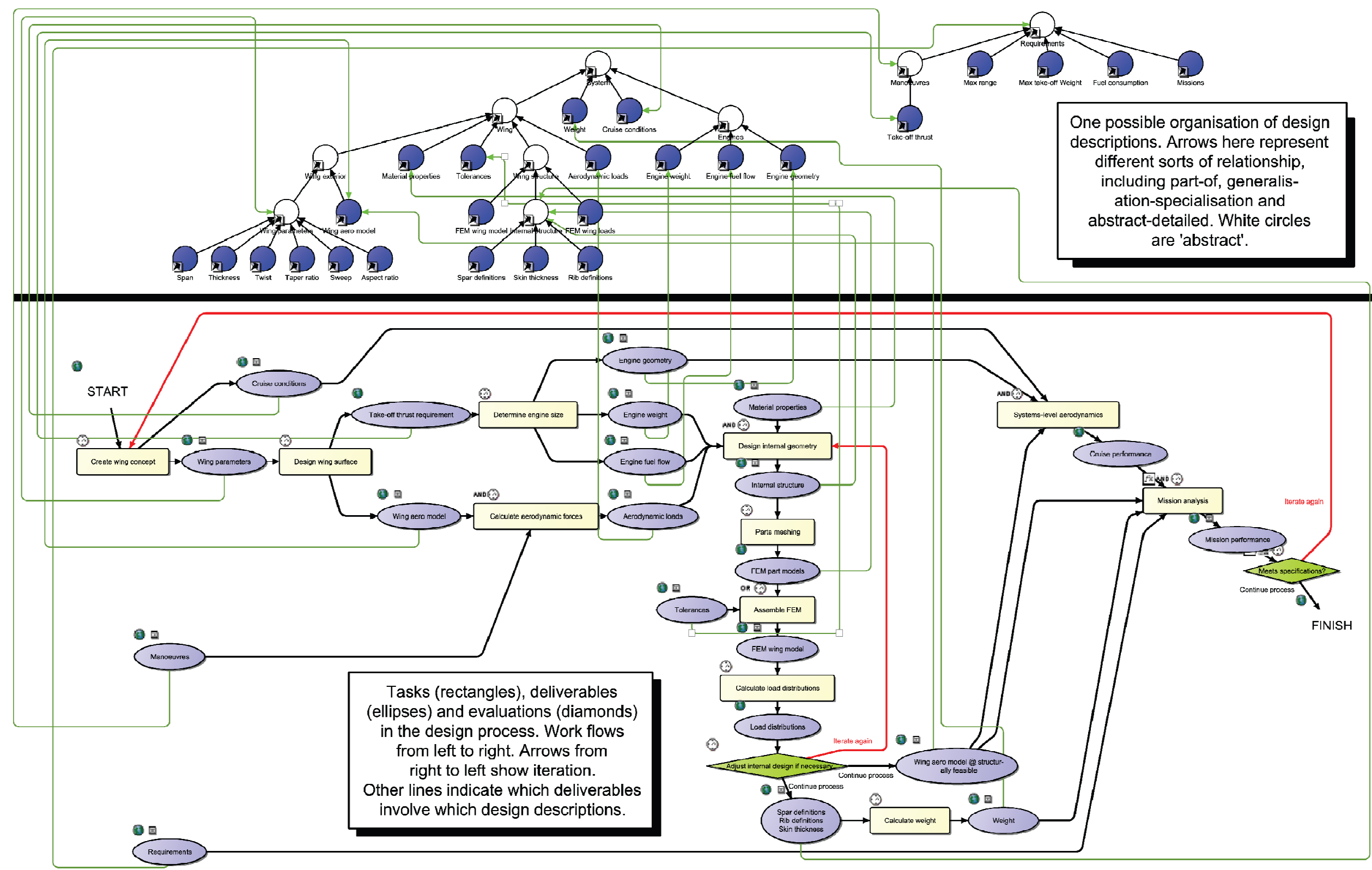

Fig. 2. Some activities, information flows and design descriptions in a generic wing design process 
Many tasks in the design process may also be undertaken using one of multiple methods. For example, early in the design process simple correlations may be used to estimate design performance. Later, when a more precise design definition is available, more sophisticated tools can be applied to perform the same task with better results. The methods used in a particular iteration of a task can also depend on the design context at that time. For instance, they can even be created on-the-fly to reflect the needs of a particular analysis and the information available when analysis is undertaken [4].

In a complex design process it can be difficult to identify and coordinate all information flows, especially those involving other sub-systems. Process participants often begin activities based upon assumptions, which may subsequently require corrective iterations when updated information becomes available. This illustrates yet another situation in which designers provide and receive information known to be uncertain, and that can cause additional complexity in the design process.

In summary, the cost and duration of the wing design process is determined by the methods that are used, the sequence in which tasks are attempted, the degree of concurrency and the strategies for managing unplanned flows, changes and iterations. The effort dedicated to tasks at each point in the process is influenced by the perceived levels of uncertainty, and consequently the dynamic organisation of the process is affected by uncertainty levels. The remainder of this paper draws on the wing design example to show that insights to support process management can be gained by modelling how processes unfold according to uncertainty within them.

\section{Uncertainty in engineering design}

There is no clear consensus or single definition of uncertainty in the engineering literature [5]. However, many definitions of uncertainty in engineering apply to "predictions of future events, to physical measurements already made, or to the unknown" [6]. In this paper, we use the term 'uncertainty' to refer to everything that contributes to a lack of definition, lack of knowledge or lack of trust in knowledge. This may differ from colloquial usage of the term but is consistent with much of the literature (e.g., $[7,8])$. Some of the main concepts found in this literature are outlined below.

\subsection{Epistemic and aleatory uncertainties}

One of the main distinctions regarding types of uncertainty in engineering design is made between epistemic and aleatory uncertainties (e.g., $[9,10])$ :

Epistemic uncertainty. Sometimes called subjective uncertainty, epistemic uncertainty is derived from ignorance or incomplete information. Some epistemic uncertainty is reducible, for instance by trade studies, measurements or consultation with experts.

Epistemic uncertainty can be viewed as arising from lack of knowledge or lack of definition. According to Hastings and McManus, lack of knowledge refers to "facts that are not known, or are known only imprecisely, that are needed to complete the system architecture in a rational way" [11]. Lack of definition reflects "things about the system in question that have not been decided or specified yet" [11]. Since they are epistemic in nature, both lack of knowledge and lack of definition may be reducible.

Similar to lack of definition, in many situations it may be difficult or impossible to reason about uncertainty because the events or parameters with which it is associated are not yet known or do not yet exist [5]. For instance, this may occur in design if the choice of product architecture 'creates' the need for certain module interface parameters.

Aleatory uncertainty. Sometimes termed objective or stochastic uncertainty, aleatory uncertainty describes the inherent variation associated with a physical system or environment - such as dimensional variation in manufactured components. Aleatory uncertainty cannot be reduced using the methods described above, although gaining knowledge about variability may allow its influence to be mitigated through design of systems to be adaptable, robust, flexible, etc. [12].

\subsection{Uncertainty in information and description}

McManus and Hastings [11] discuss how uncertainty may concern information (e.g., incompleteness, inconsistency or limited measurement quality) and may also be associated with how the information is described or modelled (e.g., due to ambiguity of description, information omitted when modelling or lack of clarity in the model's scope). This classification is similar to Eversheim et al.'s [13] categorisation of uncertainty: content-uncertainty (incompleteness, imprecision and vagueness) and context-uncertainty (unreliability, invalidity and instability).

Uncertainty concerning information may be aleatory or epistemic in nature. Uncertainty associated with description is primarily a type of epistemic uncertainty that arises from assumptions or lack of knowledge while modelling [14].

\subsection{Uncertainty in abstraction and interpretation}

The quality of any description is subject to the view an observer has of the target system and any related phenomena about which judgments are to be made. This arises since the systems or phenomena cannot be considered directly by the observer [15], but are processed through conceptual apparatus that provides ways of thinking about and terminology for describing aspects of the system.

Considering this issue, Pons and Raine [8] discuss abstraction uncertainty in engineering. Abstraction uncertainty arises from how descriptions are interpreted and used by engineers. It thus depends on the subjective perceptions of process participants and can be reduced during the design process as more details become known. The concept of uncertainty in abstraction also has implications regarding how uncertainty can be described and analysed. There is no general approach to representing and reasoning about the uncertainty associated with a design but rather several different 
methods [15]. For example, probability theory provides concepts such as variance to describe uncertainty levels whereas fuzzy set theory provides membership functions. Smithson [7] provides a taxonomy of formalisms for handling uncertainty in design according to the sources of ignorance.

However, no single method is able to model all types of uncertainty equally well [7]. The most appropriate approach should be determined by the modelling context. This includes the causes of uncertainty; how uncertainty is understood; the available information; and the purpose for modelling.

\subsection{Uncertainty associated with complexity}

Uncertainty is also associated with the complex system of interrelated models found in design [3]. Uncertainty levels associated with different descriptions of the emerging design evolve in an interdependent way, because the descriptions are related to one another through the structure of the design process system within which they are embedded.

Building on the analysis of complexity in design developed by Earl et al. [3], we consider the structure of information use in design to comprise: (1) the decomposition of the design into the abstract structure of information that defines it; (2) the representation of this abstract information as multiple, overlapping descriptions that exist as objects or data in the real world; and (3) the behaviour and organisation of activities that create and manipulate descriptions during design. In following sub-sections we argue that interdependencies between uncertainty levels depend upon this system structure, and that it also creates sources of uncertainty that impact upon design descriptions and their interpretation (Fig. 3).

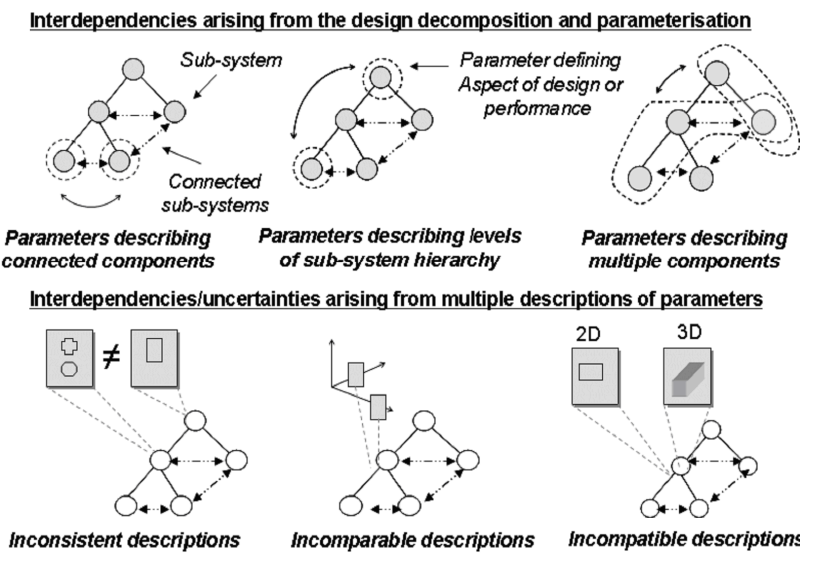

Uncertainties arising from the process of developing design descriptions
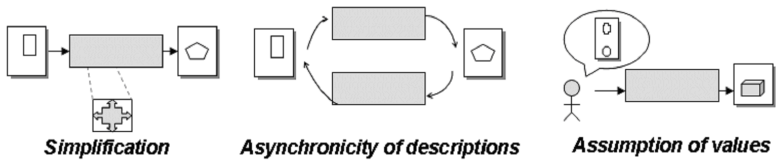

Fig. 3 A framework of factors that cause dependencies between uncertainty levels associated with design descriptions and that cause additional uncertainties in the context of the design process.

\subsubsection{Interdependencies between uncertainty levels arising from the design decomposition and parameterisation}

During design, a system is decomposed into sub-systems, components and the parameters that define them. In this context, we consider a design parameter not to be limited to numeric values such as 'radius', but to refer to any aspect of the system definition, including aspects such as geometry and material selection. We also consider performance parameters, e.g., 'weight' or 'drag', whose values cannot be defined by the designer, but can only be estimated or measured once design parameters have been specified.

Three forms of interdependency between the uncertainty levels associated with parameters arise from the structure of the design decomposition:

Interdependencies between uncertainty levels in parameters associated with interconnected subsystems or components. The levels of uncertainty associated with parameters defining connected subsystems or components are related to one another. For instance, there is an interface in the wing between the skin and ribs. The thickness of the skin impacts significantly on the design of the ribs. Consequently, rib geometry cannot be determined precisely unless the skin thickness is also precise, and vice versa.

Interdependencies between uncertainty levels in parameters associated with different levels of the system. The decomposition of a system into sub-systems and components results in parameters defining similar aspects at different levels of this hierarchy. These parameters are interdependent as they define the same aspect of the design, but are associated with different levels of its decomposition; thus the levels of uncertainty associated with them are also related.

For instance, one performance parameter in aircraft wing design is the weight of the assembled wing, which can be computed from the definitions of skin, ribs and other components. Uncertainty levels in any calculation of the wing's weight are thus directly related to uncertainty levels in the parameters defining its constituent components.

Interdependencies between uncertainty levels in parameters associated with multiple components and sub-systems. Because the decomposition of a design into parts and subsystems is determined by the way the product is designed and manufactured, many important parameters are not localised within a single component or sub-system. The uncertainty levels in parameters may thus be interdependent if those parameters are associated with any of the same parts.

For instance, during wing design the maximum bending load on the structure (a high-level performance parameter) is influenced by the geometry of all the constituent components (defined by lower-level design parameters). This implies that the uncertainty associated with any calculation of wing bending load is related in a potentially complex way to that associated with the component definitions. 


\subsubsection{Interdependencies and additional uncertainties arising from multiple descriptions of parameters}

Information is not modified directly while designingthe process operates upon multiple descriptions or models, each of which represents one or more parameters. If the scopes of several descriptions overlap, the uncertainty levels associated with them will thus be interdependent.

Uncertainty also arises from how parameters are represented during the process. In particular, use of multiple representations and multiple forms of representation introduces variation in how the design is perceived:

Uncertainty arising from inconsistent descriptions. Descriptions used during design often embody different values of the same information. This can introduce uncertainty if designers must make decisions based on inconsistent representations, or if different designers knowingly or unknowingly work with inconsistent representations. In practice, inconsistency is often inevitable and can be managed if its possibility is recognised. For instance, models that are considered old in terms of the 'iterative speed' of the process are often not trusted, and can be checked prior to use. On the other hand, unrecognised inconsistency can lead to mistakes that are integrated into the design and may require expensive rework to correct later.

Inconsistency may arise from the different types and formats of representation used during design as described below, or from the complexity of the design process and need to make assumptions, as discussed in Sect. 3.4.3.

Potential for inconsistency arising from incomparable descriptions. Any aspect of the design may be simultaneously represented in several different formats suitable for different tasks. For instance, consider the concurrent representation of geometry in data formats used by different tools. If the formats are not possible to compare using the available tools, inconsistency may exist but may not be recognised-or the recognised potential for inconsistency may lead to lack of trust in the data.

Potential for inconsistency arising from incompatible descriptions. Different tasks consider descriptions that represent the design definition in different levels and forms of abstraction-such as 2D geometry versus 3D geometry. Such models can be 'incompatible' in that they omit significant and different aspects of the design definition that is represented, again leading to potential for inconsistency. Similar issues occur when representations of the design are viewed or interpreted in different ways, or when different aspects are considered important by different stakeholders.

\subsubsection{Interdependencies and additional uncertainties arising from the process of developing design descriptions}

Since descriptions are processed by activities in the design process, the uncertainty levels associated with descriptions created by a task are related to those associated with descriptions used as input to that task. For instance, analysis based on design descriptions that are believed to be out-of-date will lead to results that are also not trusted. Descriptions are also interrelated indirectly through the process network, since analysis results influence design decisions that are subsequently integrated into the workflow. For instance, levels of uncertainty in many downstream descriptions may increase if the information on which an analysis was based is subsequently changed.

In addition to these interdependencies, the properties of each task and the organisation of the process introduce further sources of uncertainty:

Uncertainty arising from models and simplifications. Many design and analysis tasks use a physics model to derive descriptions of performance parameters from descriptions of design parameters. Assumptions in these models introduce uncertainty in analysis results additional to that which is propagated from the input. For instance, CFD models may use simple approximations in place of sophisticated turbulence models. Geometry is also often simplified and defeatured in different ways when preprocessing for different analysis tools, objectives and boundary conditions. Ordaz-Hernandez et al. [16] discuss how model simplifications should be considered according to the modelling context; trade-offs may be required between accuracy and speed of the user interactions required to perform the task.

Potential for inconsistency arising from the complex organisation of activities. Design processes are organised into concurrent streams of work that are complex and difficult to understand in their own right, and this complexity is exacerbated by the iterations that occur within and across workflows. Due in part to this complexity, participants often have limited overview of information flows and dependencies in the process. As a result descriptions and models used during design may become desynchronised and inconsistent.

Potential for inconsistency arising from introduced assumptions. The dependencies between design parameters constrain the order in which they can be determined, such that a given parameter cannot be finalised until all the values on which it depends have also been determined. In practice, there are often many cyclic dependencies between parameters, so that it is not possible to identify a sequence of determination. Assumptions must then be made about the values of parameters and design must proceed knowing that later rework may be required. Similarly, the need to divide work among many participants leads to artificially concurrent work streams with limited information flows between them, necessitating additional assumptions and potential for inconsistency that may or may not be recognised.

\subsection{Uncertainty associated with lack of trust in knowledge}

The previous subsection explains how designers must cope with an overabundance of information, including conflicting or difficult-to-compare representations of the same systems or phenomena. As a result, stakeholders may be uncertain about an issue because they don't trust the available information. For instance, an engineer may 
not trust certain information if they don't know who generated it; if that person is inexperienced; if they don't know when the information was generated; or if the information is considered old enough that it could need updating.

\section{Modelling the evolution of uncertainty during design}

Task-based models offer a way to analyse the evolution of uncertainty levels during design, because they capture the progression of distinct activities over time, along with the creation and modification of inputs and outputs.

Most task-based design process models in the literature are based on graphical activity networks (e.g., [17]) or Dependency Structure Matrices (e.g., [18]). These models assume that once the input information for a task is known, the output information can be calculated or deduced and the next task may be identified and attempted in turn. Some task-based models allow simulation of a process; in these approaches, the duration of each task is generally modelled either as a precise value or as a probability density function. Such models can be used to investigate the impact of uncertainty on project performance - for instance, by exploring the situations in which delays encountered while completing certain tasks will propagate to the end of the process. However, they do not represent the evolving uncertainty associated with the design itself.

Other task-based models do relate aspects of productrelated uncertainty to process behaviour. For instance, Krishnan et al. [19] model situations in which tasks release preliminary outputs to their successors before work is finalised. They consider preliminary releases to be intervals within which the final value must lie. Simulation models that consider product-related uncertainty include Signposting [20] and the adaptive product development process (APDP) [21]. These approaches qualify the information that tasks require and produce by describing different aspects of its maturity. In Signposting, a single item of maturity metadata described as 'confidence' is associated with each design parameter, used to represent designers' belief in the quality of information. In the APDP model, the meta-data relates to the risk that the design will not meet technical performance measures (TPMs). Both models assume that tasks are attempted when sufficient maturity is reached in all inputs. Completing a task causes maturity in its outputs to increase, or to reduce if the task reveals a need for rework.

These models focus primarily on the effect of maturity levels on task sequencing and on the appropriate choice of methods to perform each task. However, in design-byanalysis processes such as wing design, the sequence of tasks is largely fixed by data flow constraints between design tools. We therefore seek to model how the iterative dynamics of the process vary given a fixed structure of activities, and how this is governed by evolving uncertainty levels. Furthermore, existing approaches do not support modelling the different forms of uncertainty discussed in Sect. 3.

\subsection{A task-based model considering uncertainty levels}

An enhanced task-based modelling approach was developed to study the impact of evolving uncertainty levels on the design process. The approach is based on a graphical Input-Process-Output notation in which each task produces deliverables that are consumed by successors. The properties o each task are specified as functions of the uncertainty levels associated with input descriptions. Task properties treated in this way are (1) duration; (2) propensity to generate iteration; and (3) uncertainty levels associated with the output descriptions generated by the task. Individual task definitions, such as that shown in Fig. 4, enable a process network, such as that shown in Fig. 2, to be simulated taking into account uncertainty levels and their evolution. The remainder of this section details the new model by considering the following questions:

- Which aspects of uncertainty should be considered?

- How should uncertainty levels be represented?

- How should the tasks' properties be modelled?

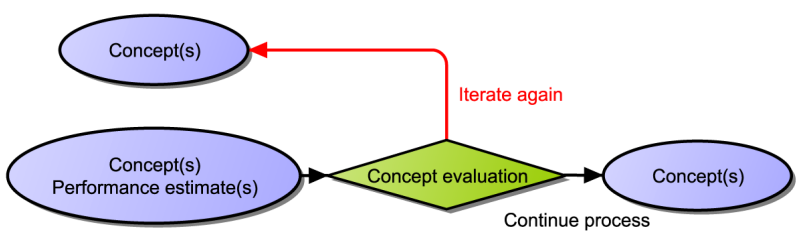

Fig. 4. Example task definition

\subsection{Which aspects of uncertainty should be considered?}

Drawing on the literature and analysis in earlier sections, five aspects of uncertainty were identified for inclusion in the model (Table 1). Each of these aspects was chosen because it is influential upon iterative behaviour - a key issue that is considered in almost all design process models [23]. The five aspects and examples of how they influence the design process are discussed below.

Imprecision. Early in the design process, a wide space of possible solutions is kept in mind by the designer. For instance, in wing design it is not possible to precisely specify geometry until load cases are precisely defined. In the case study, engineers communicated imprecise loads by specifying interval values (about $\pm 15 \%$ at the beginning of embodiment design).

Imprecision arises when design decisions remain open, and many authors therefore argue that managing imprecision is important during design. For instance, Eckert et al. [32] describe how design margins may be maintained during complex collaborative design to allow headroom for absorbing changes that may arise later. Similarly, Sébastian and Ledoux [25] discuss the use of imprecise domains to represent emerging solutions in solvers for constraint satisfaction problems in preliminary design (eg., [26]). Antonsson and Otto [27] introduce the Method of Imprecision, showing how fuzzy sets can be used to reason about imprecision during design. 
Table 1. Levels of up to five aspects of uncertainty may be associated with each description in the model

\begin{tabular}{|c|c|}
\hline Name & Description and example of use in the model \\
\hline Imprecision & $\begin{array}{l}\text { Description: The 'range' within which information could lie [24]. As design decisions are made imprecision } \\
\text { tends to progressively reduce until a final, precise value is determined [27] } \\
\text { Example: Greater precision justifies application of more sophisticated design tools and methods, which may } \\
\text { require progressively greater effort [28] }\end{array}$ \\
\hline Inconsistency & $\begin{array}{l}\text { Description: Different descriptions provide inconsistent views of the same design parameters } \\
\text { Example: Potential for inconsistency is introduced by tasks that make assumptions to resolve cyclic } \\
\text { dependencies or to perform work concurrently [29]. Rework must be undertaken to reconcile inconsistency } \\
\text { when revealed by integration or evaluation tasks }\end{array}$ \\
\hline Inaccuracy & $\begin{array}{l}\text { Description: Expected discrepancy between the predicted values of performance parameters and their actual } \\
\text { values, which could be estimated from evidence gained through tests [24] } \\
\text { Example: As more sophisticated analysis methods are brought to bear during the design process they lend } \\
\text { progressively greater accuracy to the estimated design performance [30] }\end{array}$ \\
\hline Indecision & $\begin{array}{l}\text { Description: A range of alternative options may be represented or held in mind. Qualitatively different to } \\
\text { imprecision as the extent of each option may be small while the range of the entire selection may be large } \\
\text { Example: Set-based design advocates considering multiple options during design, with progressive } \\
\text { convergence towards a single option [34]. The duration of many tasks may depend on the number of, and } \\
\text { extent of differentiation between the options }\end{array}$ \\
\hline Instability & $\begin{array}{l}\text { Description: More unstable descriptions are more likely to change } \\
\text { Example: Instability may be increased by events that increase the likelihood that rework will be required; for } \\
\text { instance, the receipt of a change request could increase expected instability in many design descriptions, } \\
\text { which may require knock-on change [32] }\end{array}$ \\
\hline
\end{tabular}

Inconsistency. As discussed in Sect. 3.4.3, inconsistency between the different descriptions used during design may arise from several sources including assumptions made in the design process, simplifications in modelling and lack of overview. The potential for creating inconsistency should be considered in cases where multiple descriptions of the same information are created; where simplifications or transformations of models are made; or where work is divided into concurrent streams. Inconsistency may be revealed by integration or testing tasks, requiring iteration to correct.

(Expected) Inaccuracy. Design involves tools and methods that lend increasing levels of fidelity to the information they create [28]. For instance, the fidelity of preliminary aeroengine gas path design can be quantifiably related to the tools used at each step in the design process [30]. Designers can thus assess the accuracy of their performance estimates with respect to the values that might later be determined through experiment or more accurate analysis methods. The inaccuracy believed to be associated with particular descriptions reflects designers' confidence in their estimates and thus the effort they will dedicate to refining the design at any point in time.

In our model, expected inaccuracy aggregates many sources and types of uncertainty discussed in Sect. 3, such as simplification in calculations and uncertainty associated with absence of data. The concept of expected inaccuracy used here should be distinguished from actual inaccuracy and from insufficiency - both of which we view as process information rather than aspects of uncertainty. Actual inaccuracy refers to the difference between a given estimate and an actual value, whereas insufficiency refers to the inability of the design to deliver required performance [7]. Expected inaccuracy may be revealed as insufficiency and/or actual inaccuracy through test or analysis.

Indecision. When a satisfactory solution cannot be identified by sequential execution of tasks, an iterative process must be used to converge upon a solution [28].
This typically involves both divergence and convergence of the solution space [17]. When the solution space diverges, designers hold in mind multiple alternatives which are subsequently evaluated prior to selecting the most promising to take forward. Indecision between alternatives is used in our model to represent this. In general, the greater the indecision associated with a description, the more time a task that requires consideration of all the options is likely to take.

Instability. The instability associated with design descriptions can influence how designers approach their tasks. For instance, if every designer spends little effort on their tasks because they believe the input information is likely to change, many fast iterations will be required to complete the design. In contrast, if assumptions are not expected to change, the process may be organised into a smaller number of more time-consuming iterations. This may incur lower co-ordination overhead, although it may also result in additional rework if change is required. The process may also be less agile, as infrequent information transfer suggests there are fewer opportunities to recognise and respond to problems and opportunities that arise during design. Events such as the decision to freeze aspects of the design or the receipt of a change request may impact upon levels of perceived instability and thus influence the dynamic structure of the design process.

In summary, each design description incorporated in the model may be qualified with the levels of one or more of these five aspects of uncertainty. The levels that should be modelled for a given description will depend upon the context in which it is used-in general, only information that is progressively developed and involved in design iteration need be considered. Apart from design descriptions, uncertainty levels may also be associated with other information or knowledge that influences the design process. For instance, it may be appropriate to model the uncertainty associated with aspects of design space understanding, because understanding impacts on the quality of decisions and can thus affect the rate of design convergence. 
Table 2. Extreme values for each uncertainty level

\begin{tabular}{lll}
\hline Uncertainty level & Minimum value & Maximum value \\
\hline Imprecision & 0 Defines a point value or single concept & 1 Encompasses all conceivable content \\
Inconsistency & $\begin{array}{l}\text { 0 Known to be completely in accord with } \\
\text { other descriptions }\end{array}$ & 1 Known to be completely inconsistent \\
Inaccuracy & 0 As accurate as possible & 1 No confidence in accuracy \\
Indecision & 1 A single option is represented & $\infty$ All options are represented \\
Instability & 0 Never expected to change & 1 Expected to change immediately \\
\hline
\end{tabular}

\subsection{How should uncertainty levels be represented?}

As discussed in Sect. 3.3, uncertainty can be represented using various approaches including probability theory, fuzzy set theory, evidence theory and possibility theory. Due to its level of abstraction, the task-based model proposed here does not represent the content of design descriptions - but only the context in which they are used, i.e., the tasks that require and produce them. Thus, the aforementioned approaches to represent and reason about uncertain information are not easy to apply. We therefore model uncertainty 'levels' without any reference to the 'content' of information (the 'content' of a numeric parameter, for instance, is its actual value). Each level of uncertainty associated with a design description or other information in a model is described as a real number. Interpretations for the extreme values are given in Table 2 .

\subsection{How should the tasks' properties be modelled?}

For each task, its duration, the probability of each outcome occurring, and the effect of each outcome on uncertainty levels must be considered to determine how they vary with respect to uncertainty levels in the task's input(s). The suggested approach is to formulate a qualitative explanation for the task's operation, then translate this into algebraic expressions.

To illustrate, imagine that the task in Fig. 4 represents a stage-gate at which a number of design concepts are evaluated. Within this hypothetical task, some of the candidates may be immediately eliminated. One of two outcomes then occurs: the remaining concepts may be passed forward to the next step; or are passed back for revision. This could be modelled as explained below and detailed in Table 3:

- Duration varies in proportion with indecision, because more time is required to consider more concepts.

- Rework probability depends on indecision in concepts and inaccuracy in performance. If more concepts are evaluated, there is a higher probability that at least one will meet the evaluation criteria. On the other hand, the more inaccurate the performance estimate, the less information is available to justify further refinement of the concepts. Hence, the rework probability reduces as indecision and inaccuracy increase.

- Output uncertainty levels are mapped from input levels depending on which outcome is selected. In this case, the output mappings are assumed to be independent of outcome. High accuracy of input allows more concepts to be eliminated on a given iteration. Therefore, indecision in the output concept depends on indecision in the input concept and on inaccuracy of the input performance. Other uncertainty levels remain unchanged.

To develop functions that express the qualitative explanations, it is useful to consider a specific context in which the task might be executed and pose the following questions: What would the uncertainty levels be in that context? What should the resulting task properties be? How would they then vary with changes in each input level? Which level(s) have greater effect? Are the effects linear? What are the extreme values for each property?

A spreadsheet can be used to verify that each function gives the desired result under different combinations of the input levels. For task definitions involving interacting effects, as in this case, several iterations may be required to refine the definition. Many tasks might not be significantly affected by uncertainty levels, in which case their properties may be modelled as point values or probability distributions. Only those tasks involving process-related decisions, such as whether to revise concepts or which methods to apply, are likely to require complex mappings such as those in Table 3.

Table 3. Property definitions for the task of Figure 4

\begin{tabular}{|c|c|}
\hline Property & Definition \\
\hline Duration & $3 \times$ Indecision $_{\text {in }}$ \\
\hline Rework probability & Indecision $_{\text {in }}^{-1} \times 0.8^{\text {Inaccuracy }_{\text {in }}+1}$ \\
\hline Output levels: Concept & (mappings for all outcomes) \\
\hline Imprecision $_{\text {out }}=$ & Imprecision $_{\text {in }}$ \\
\hline Inconsistency $_{\text {out }}=$ & Inconsistency $y_{\text {in }}$ \\
\hline Inaccuracy $_{\text {out }}=$ & Inaccuracy $_{\text {in }}$ \\
\hline $\begin{array}{l}\text { Indecision }_{\text {out }}= \\
\text { Instability }_{\text {out }}=\end{array}$ & $\begin{array}{l}\max \left(1, \text { Indecision }_{\text {in }} * \text { Inaccuracy }_{\text {in }}\right) \\
\text { Instability }_{\text {in }}\end{array}$ \\
\hline
\end{tabular}

\section{Illustrative applications}

To illustrate some of the possibilities for analysis based on this approach, two models were developed to explore the impact of uncertainty levels during design.

\subsection{Analytic model}

The first application builds on a high-level generic process model (Fig. 5) to consider two questions pertinent to many design-by-analysis processes:

How much effort should be spent on concept analysis? Much of the performance of a design is 'locked in' when high-level parameters are frozen during concept design [30]. More time spent on analysis of 


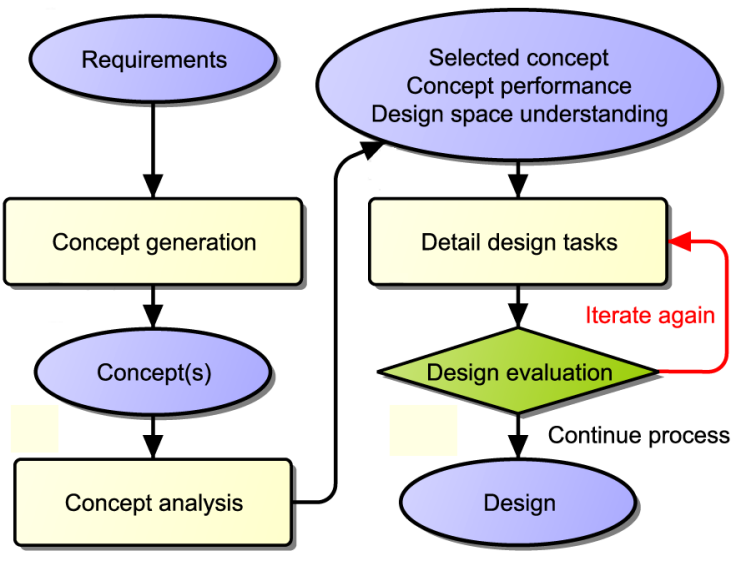

Fig. 5. Generic design process used in the analytic model

concepts creates more accurate information with which designers can judge the emerging design. This could allow refinement of the high-level parameters and reduce the likelihood of expensive iterations during detail design. On the other hand, more concept analysis increases overall effort.

How many feasible concepts should be considered during early design? In many design-by-analysis processes, alternative concepts are eliminated very early. This can limit consideration of innovative designs that potentially offer better performance [22]. Some authors propose using intermediate-fidelity tools that allow more concepts to be taken forward further into the process [33], enabling application of set-based design principles [34]. By allowing a wider space of distinct alternatives to be considered, this could increase the likelihood of finding a good concept and reduce the probability of iterations later. Considering and analysing alternatives could also help develop design space understanding, which we define as the knowledge that enables designers to make good design decisions without requiring extensive analysis first. This can help to more quickly converge on a solution if iterations do occur [30]. However, taking multiple alternatives forward also incurs additional effort.

An influence network was formulated to show these factors and interactions (Fig. 6). By considering the qualitative description above and the information flows in Fig. 5, equations were written to define each relationship (Table 4). The equations were combined to eliminate model-internal parameters and show the impact of concept analysis effort and the number of concepts considered on expected process duration. The result was evaluated using a spreadsheet for different values of the process-specific constants, and the equations were iteratively adjusted until all parameters varied in accord with expectations.

The results from two configurations are shown in Fig. 7. This illustrates that low overall duration $\mathrm{k}$ may obtained through low concept analysis effort a applied to a moderate-to-high number of concepts $b$, or high effort applied to a single concept. Comparison of the two configurations implies that taking several feasible concepts forward is a more attractive proposition in processes where the duration of a detail design cycle is

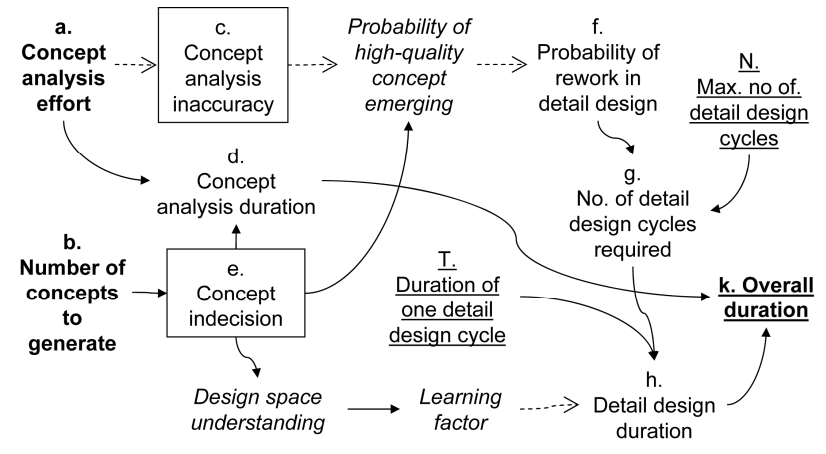

Fig. 6 Parameters and relationships studied in the analytic model. Dashed arrows show negative relationships where a decrease in the upstream parameter creates an increase in the downstream parameter

high relative to the effort spent analysing each concept. This is because the effort for analysing additional concepts is overwhelmed by the benefits of reducing iteration probability and cycle time. More trials using different combinations of the process-specific constants showed that the shape of the response surface remains similar, although the peaks and troughs are emphasised differently.

This analysis is deliberately simple, intended to illustrate model development in a reproducible way. The model could be further developed to give more realistic results. Nevertheless, even this simplified situation shows how modelling uncertainty levels can help develop qualitative insights into design process decisions and their effects.
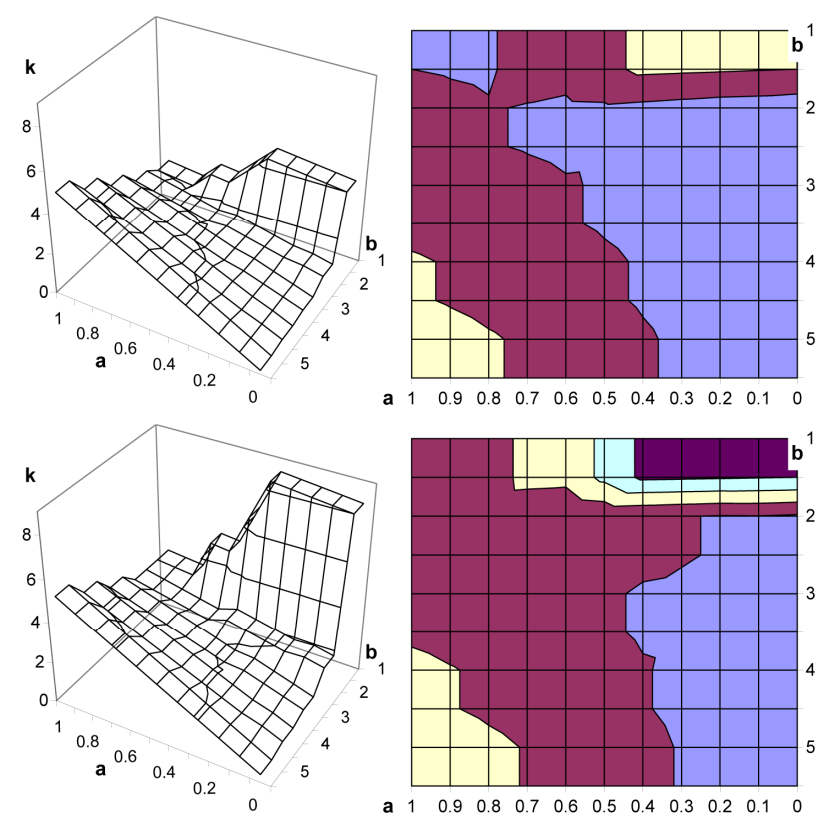

Fig. 7 Two configurations of the analytic model, showing $\mathrm{N}=$ $3, \mathrm{~T}=1$ (top row) and $\mathrm{N}=3, \mathrm{~T}=2$ (bottom row). Each righthand plot shows lines of constant height on the left-hand plot in the same row

\subsection{Simulation model}

The equations in Table 4 were straightforward to formulate and solve because the situation contains no cyclic dependencies between variables, no complex 
Table 4. Definitions for the relationships shown in Fig. 4

\begin{tabular}{ll}
\hline Definition & Rationale \\
\hline$k=d+h$ & Overall duration $k$ is sum of concept phase duration $d$ and detail phase duration $h$ \\
$d=a \times b$ & Concept phase duration $d$ is proportional to effort per concept $a$ and no. of concepts $b$ \\
$h=T \sum_{n=1}^{g} b^{-(n-1)}$ & Baseline duration $T$ reduces by learning factor that is inv. prop. to $b$, on each of $g$ cycles \\
$g=\sum_{n=1}^{N} \operatorname{ceil}\left(\max \left(f^{n}-0.2,0\right)\right)$ & Detail design will be complete in $g$ cycles with $80 \%$ confidence, max. $N$ cycles allowed \\
$f=(1-c) \times b^{-1}$ & $\begin{array}{l}\text { Probability } f \text { of detail design rework is proportional to concept analysis accuracy }(1-c) \\
\text { and inv. proportional to no. of concepts considered } b\end{array}$ \\
$c=a^{0.5}$ & Increases in effort $a$ give diminishing returns to accuracy
\end{tabular}

The independent variables are $a$ and $b$. The dependent variable is $k$. Process-specific constants are $T$ and $N$ (also 0.2 and 0.5 ). All other parameters are internal to the model

flows between tasks, no resource dependencies, and because uncertainty levels do not change over time.

The wing design process model shown in Fig. 2 was revisited to show how discrete-event simulation can more closely incorporate the features and complexity of a reallife process. Most of this diagram maps to the right-hand column of Fig. 5. To configure the more complex model for simulation, the first step was to identify the main factors that drive iterations. To do this we drew on the work of Jarrett et al. [31], who explain how each method used in a design-by- analysis process is suitable for a given point in the design process, because it requires certain confidence in input data and confers a particular level of accuracy on the solution.

For a particular set of analyses, the candidate design undergoes iterations to consider how changes impact a particular set of objectives. During iterations, performance levels can remain 'stagnant' but understanding about the design space is progressively generated. Eventually, the improved understanding allows designers to make a change that creates a 'surge' in design performance [31]. For the next iterations, additional objectives may be considered and more accurate methods may be used.

To model this situation, the wing design process was considered to comprise an execution process nested within a management layer, and to execute in cycles. The management layer defines the work that will be performed on a given cycle. These instructions are passed to the execution process, which determines how long the work will take. The execution process will repeat until enough design space understanding is generated to meet the specified objectives. Control is then passed back to the management layer, which sets up the next cycle. This model is shown in Fig. 8. Our modelling software allows the central box to be expanded to show the detail of Fig. 2.

\subsubsection{Modelling the management of iterations}

The uncertainty levels considered in the management layer are (required) inaccuracy of design analysis and indecision in design objectives. In overview, the model begins with indecision of design objectives $=1$, denoting that a single objective is considered. Within the topmost task of Fig. 8, design analysis inaccuracy is set to depend directly on this value. The model thus assumes that as more objectives are considered, higher-fidelity methods will be brought to bear. A cycle of the execution process then occurs, influenced by the inaccuracy and indecision levels that were set. When the execution process is complete, the task at the bottom of Fig. 8 requires that high-level iterations continue unless all objectives have been considered and low inaccuracy has been reached. If iterations are to continue, the indecision in design objectives is increased and the cycle begins again. The amount at which indecision in objectives is increased represents the planned progress rate, similar to schedule pressure. A higher value implies that designers would be asked to complete more work on each cycle. Planned progress rate is constant during a given simulation run, and is the main independent variable in this example model.

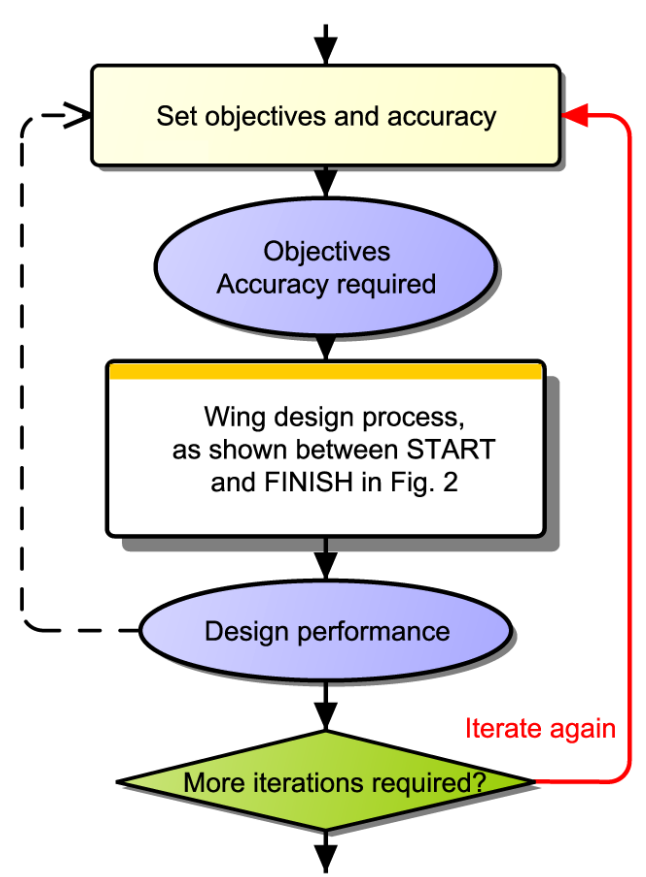

Fig. 8 Management layer of the wing design process model. The dashed arrow shows a feed-forward into the next iteration 


\subsubsection{Modelling the execution of iterations}

The behaviour of the execution process is governed by the inaccuracy of design space understanding as well as the two uncertainty levels discussed above. The first task in Fig. 2 receives these uncertainty levels directly from the management layer. These affect its outputs which are passed to its successors, and so on.

Each task in Fig. 2 was considered to determine how its properties would change according to its input uncertainty levels. Higher levels of input indecision generally require more time for design tasks because all the issues must be considered by a designer. Lower levels of input inaccuracy require more time for analysis tasks, because computationally-expensive methods must be used.

In reality, tasks that determine high-level parameters are completed early in the process. We thus configured these tasks to be executed only when inaccuracy of design analysis is higher than a specified value. Certain descriptions and their uncertainty levels are therefore progressively 'frozen' once certain levels of inaccuracy are reached. Those levels are then passed forward to all future iterations. Similarly, some analysis tasks are only attempted when the inaccuracy is low.

At the end of an execution process cycle, inaccuracy in design space understanding is reduced. A formula was defined assuming that design space understanding relevant to meet the current objectives is a sum of 'general design space understanding' and 'problemspecific design space understanding'. The former increases slowly, according to the number of execution iterations undertaken in total until that point. The latter increases more rapidly, according to the number of iterations undertaken so far within the current cycle. Inclusion of the 'general design space understanding' ensures that all cycles derive some benefit from the knowledge gained in prior explorations. This is similar to the learning factor incorporated in the analytic model discussed previously.

If the design space understanding reaches the required accuracy of design analysis, the execution process has met its objective and passes control back to the management layer. Otherwise, the execution process will be repeated.

\subsubsection{Simulation}

A discrete-event Monte-Carlo simulation algorithm based on the Applied Signposting approach [17] was used to evaluate the model. In overview:

- All tasks for which every input exists, at least one input is marked as 'recently updated', and for which no 'upstream' work is pending, are identified to start.

- The duration of each task thus identified is determined according to the uncertainty levels in its inputs. The task's inputs are each marked as 'already considered'.

- The simulation clock is advanced to the next time at which one or more tasks are marked for completion. If no such tasks are found, the algorithm terminates.
- Each completing task is considered. If that task has multiple outputs, a single output is chosen according to the task definition and uncertainty levels in inputs. All deliverables for the selected output are marked as 'recently updated', and their uncertainty levels are updated as specified in the task definition.

- $\quad$ The first step is revisited.

Simulation creates a profile of possible processes, each indicating which tasks were in execution in which times and how the uncertainty levels evolve. Alternative model configurations may be generated and analysed to explore many different issues relating to the process and its management.

\subsubsection{Illustrative analysis}

In our example model, the planned progress rate determines how quickly analysis of the different design objectives should be factored into the process. If planned progress rate is very high, many objectives are considered from the outset of design. The model then implies that design space understanding may initially be insufficient to make decisions that meet those objectives. Effort-intensive iterations considering the multiple objectives will then be required to build up the understanding. On the other hand, if the various objectives are incorporated too slowly, effort may be wasted if each iteration of the execution process is not used to full capacity.

Two simulated processes that result from different planned progress rates are shown in Fig. 9. Because each task in the model represents an activity that can be undertaken using one of multiple methods and applied to a number of objectives, these Gantt charts represent 'rolled up' versions of the plans that might be used in practice. Hence, a great many iterations appear for each line in the Gantt charts. Tasks to the left of Fig. 2 appear in the upper rows of the Gantt charts. For a particular row, a dense area of bars indicates that the task was a focus of iterations at that point in time.

Comparing the two Gantt charts illustrates that, when the planned progress rate is increased, rapid progress is indeed made early on-yet significant delays are caused later. The optimal planned progress rate was located by varying its value, simulating the process, and considering the impact on overall duration (Fig. 10).

In summary, this example illustrates how potential tradeoffs and improvements in a process can be identified by considering the role of uncertainty levels and preparing a model for simulation. In the example, the degree of importance of the trade-off and the location of the optimal point emerges from the configurations of tasks within the execution process, and the way that uncertainty levels consequently evolve within the simulation. The example model thus combines a highlevel, top-down explanation of the process' behaviour with a low-level, bottom-up representation of how individual tasks respond to changing uncertainty levels. Interaction between these levels of representation drives complexity in the model and required use of simulation to create and explore it. 


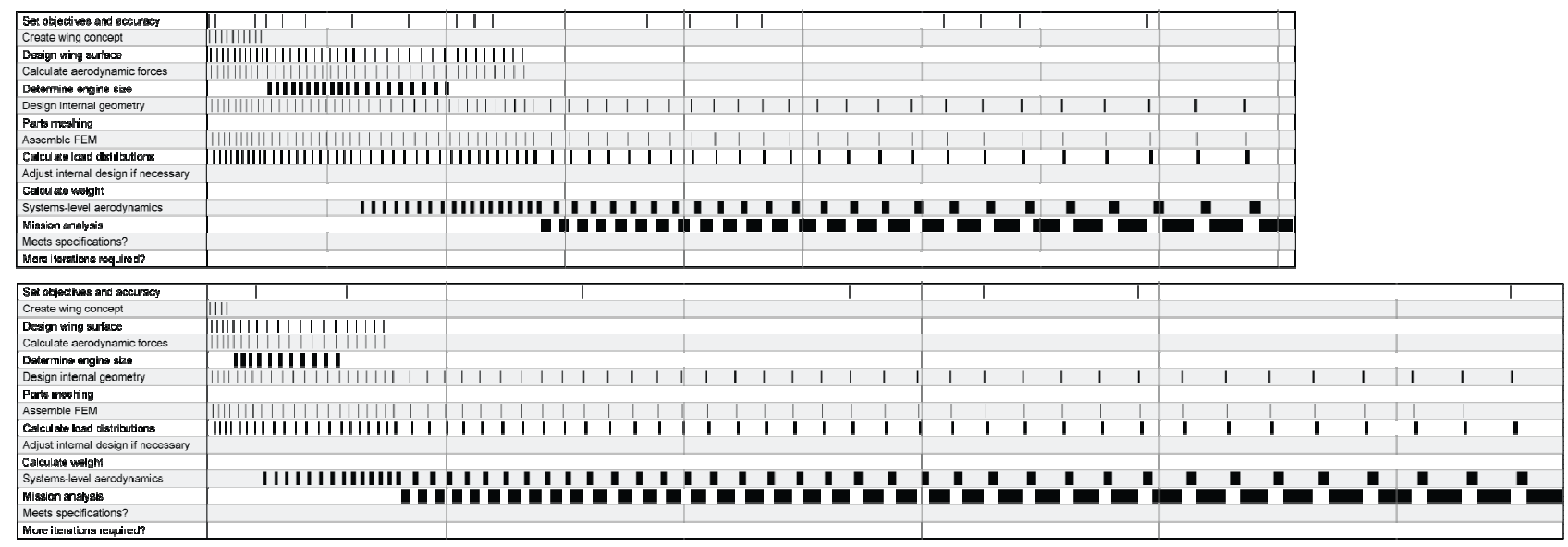

Fig. 9 Impact of planned progress rate on the unfolding process. The top chart shows the process resulting from the 'best' planned progress rate, equivalent to the low point of Fig. 10. The bottom plot shows the process resulting from a higher-than-optimal planned progress rate. Both use the same scale

\section{Discussion and outlook}

Uncertainty levels provide a way to capture some of the key aspects of design data that affect the design process as it unfolds. Through uncertainty levels, each task's outcome is dependent upon its predecessors and affects its successors.

The uncertainty levels thus cause interactions between tasks in addition to those that occur through the 'binary' requirement for information to be released from predecessors. The applications discussed in Sect. 5 have shown how these additional interactions can help explain and model aspects of iterative behaviour.

The applications also show how modelling uncertainty levels can help assess the impact of different policies for managing the design process. This approach is wellsuited to analyse potential process improvements that impinge not only on the task duration and outcome, but also on the emerging design. The models we describe were developed to illustrate the modelling process and are not intended to provide accurate results. As with any model, the quality of insights gained depend on the input data and assumptions; for practical application, these should be calibrated against historical data and/or expert opinion.

There remain many opportunities for further work to extend and apply the approach, including:

\subsection{Support for knowledge elicitation}

The model presented above requires tasks to be considered individually and in depth to determine their properties. A more rigorous and repeatable procedure for eliciting task properties would help to apply the approach in practice.

One way to approach this might be to consider generic types of task and how each is influenced by uncertainty levels. A taxonomy of generic task types and their characteristic behaviours could provide a starting point to support modelling. Categorising a specific task using the taxonomy could provide a template for defining its behaviour.

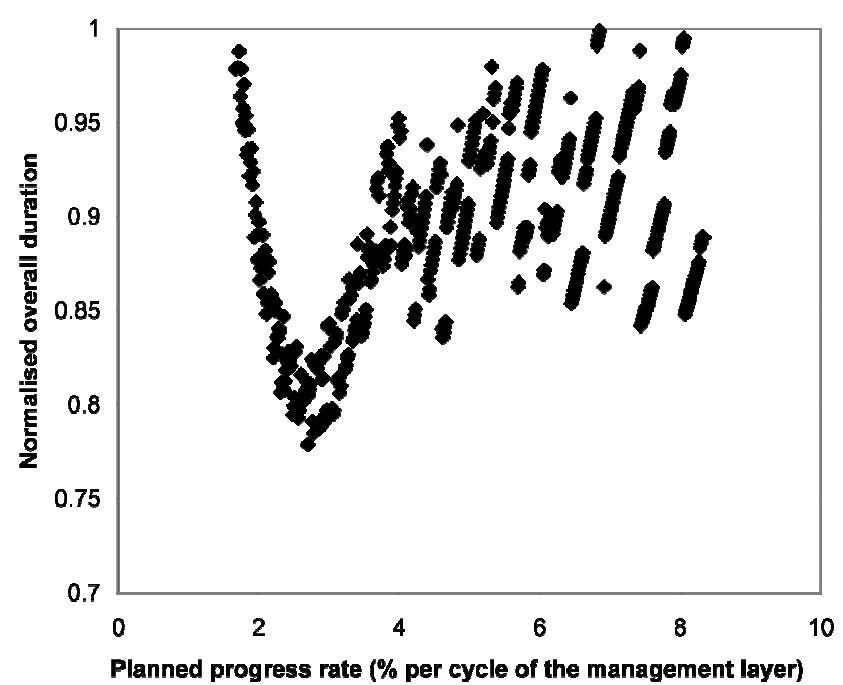

Fig. 10 Results from simulation showing the planned progress rate versus overall duration

Experiments could also be undertaken based on simple, repeatable design problems to assess the relationship between perceived uncertainty levels in task input information, the task's duration and outcome, and the uncertainty levels in outputs. This could lead to a deeper understanding regarding how uncertainty levels affect design tasks, and how this in turn influences the dynamic structure of the process.

\subsection{Monitoring project progress}

Another opportunity for further research is to explore how levels of uncertainty could help to monitor project progress.

One possibility is that simulation could be used to evaluate the sensitivity of the process to each uncertainty level. Given a particular point in the process workflow and the estimated levels of uncertainty at that time, levels might be varied and simulation used to assess the impact on total duration and risk. The sensitivity of process 
duration to change in a particular uncertainty level could correspond to the criticality of that level at that point in time.

This type of analysis could also offer opportunities to improve project performance, through methods analogous to critical path management. If critical uncertainties could be reduced, i.e., by performing additional design or analysis tasks, this might improve the performance of the project. Conversely, expending effort to reduce the levels of less critical uncertainties might be best avoided.

\subsection{Investigating iterative dynamics}

Iteration is a key aspect of the design process that makes it difficult to understand and manage [23]. Understanding the relationship between observed progress, iterations and project delivery is thus important to effective design management.

We suggest this understanding could be improved by using observations of how uncertainty levels evolve over time to reveal the behaviour of iterations - for instance, to assess whether a project is undergoing controlled refinement iteration or uncontrolled rework. To illustrate, periodic change in perceived levels of uncertainty might suggest controlled refinement iterations in which a design is repeatedly modified, analysed and improved. In contrast, irregular changes in uncertainty levels might indicate rework undertaken to correct design errors.

Such analysis could provide another indication of where effort and resources should be focused to expedite delivery. Knowing that a project is undergoing iteration to explore the design space, it might be appropriate to dedicate resource to analysing the design solutions in order to more quickly identify a feasible concept. However, if the dominant form of iteration was rework to correct errors, effort might instead be focused on the sources of low quality that initiate problems.

\section{Conclusions}

It is often very challenging to foresee the causes of delays and iterations in design. This paper has argued that iterative design processes unfold in a direction influenced by the evolution of uncertainty levels within them. Modelling the relationship between uncertainty levels and design process outcomes can help understand the causes of delays, and assist in identifying and evaluating policies for managing the design process.

The paper makes two main contributions. First, a conceptual framework highlights the different types of interdependencies between evolving levels of uncertainty in the design. Uncertainty levels evolve in a way influenced by relationships between the aspects of the design which descriptions represent, the use of multiple, overlapping descriptions during the design process, and the process of defining and refining the design.

The second contribution is to show how design processes can be simulated using task network models that take into account evolving uncertainty levels. We propose that task behaviour can be modelled in terms of the uncertainty levels associated with input descriptions as well other information and knowledge used to perform the task. The model captures the bidirectional relationship between tasks and the uncertainty levels associated with the emerging design - how the evolution of uncertainty levels depends on the design process organisation, and conversely how the process unfolds dynamically depending upon the uncertainty levels when decisions are made.

Our approach could provide a mechanism to explore the impact of product-related uncertainty on design process performance, and ultimately to better understand how such risks could be managed. However, the research reported here is in a relatively early stage, so there is still much work remaining to refine the modelling approach and explore potential applications.

Acknowledgments The authors would like to thank members of the Bath IDMRC who collaborated on the case study, as well as David Wyatt, Warren Kerley and the anonymous reviewers.

\section{References}

1. Grebici, K., Goh, Y.M., McMahon, C.A.: Uncertainty and risk reduction in engineering design embodiment processes. In: Proceedings of the 10th International Design Conference (2008)

2. Kesseler, E., Vankan, W.J.: Multidisciplinary design analysis and multi-objective optimisation applied to aircraft wing. WSEAS Trans. Syst. Control 2(1), 221227 (2006)

3. Earl, C.F., Eckert, C.M., Clarkson, P.J.: Predictability of change in engineering: a complexity view. In: Proceedings of the ASME International Design Engineering Technical Conferences (2005)

4. Balachandran, L.K., Guenov,M.D.: Computational workflow management for conceptual design of complex systems. AIAA J. Aircr. 47(2), 699-704 (2010)

5. Thunnissen, D.P.: Propagating and Mitigating uncertainty in the design of complex multidisciplinary systems. Ph.D. thesis, California Institute of Technology (2005)

6. Earl, C.F., Johnson, J., Eckert, C.M.: Complexity. In: Clarkson, P.J., Eckert, C.M. (eds.) Design Process Improvement-A Review of Current Practice, pp. 174197. Springer, London (2005)

7. Smithson, M.: Ignorance and Uncertainty: Emerging Paradigms. Springer, New York (1989)

8. Pons, D.J., Raine, J.K.: Design with uncertain qualitative variables under imperfect knowledge. Proc. Inst. Mech. Eng. Part B J. Eng. Manuf. 218(8), 977-986 (2004)

9. Oberkampf, W.L., Helton, J.C., Joslyn, C.A., Wojtkiewicz, S.F., Ferson, S.: Challenge problems: uncertainty in system response given uncertain parameters. Reliab. Eng. Syst. Saf. 85(1-3), 11-19 (2004)

10. Mourelatos, Z., Zhou, J.: Reliability estimation and design with insufficient data based on possibility theory. AIAA J. 43(8), 1696-1705 (2005) 
11. McManus, H., Hastings, D.: A framework for understanding uncertainty and its mitigation and exploitation in complex systems. IEEE Eng. Manag. Rev. 34(3), 81-94 (2006)

12. Chalupnik, M.J., Wynn, D.C., Clarkson, P.J.: Approaches to mitigate the impact of uncertainty in development processes. In: Proceedings of the 17th International Conference on Engineering Design, vol. 1, pp. 459-570 (2009)

13. Eversheim, W., Roggatz, A., Zimmermann, H.-J., Derichs, T.: Information management for concurrent engineering. Eur. J. Oper. Res. 100(2), 253-265 (1997)

14. Nilsen, T., Aven, T.: Models and model uncertainty in the context of risk analysis. Reliab. Eng. Syst. Saf. 79(3), 309-317 (2003)

15. Zimmermann, H.J.: An application-oriented view of modeling uncertainty. Eur. J. Oper. Res. 122(2), 190-198 (2000)

16. Ordaz-Hernandez, K., Fischer, X., Bennis, F.: A mathematical representation for mechanical model assessment: numerical model qualification method. Int. J. Comput. Math. Sci. 1(4), 216- 226 (2007)

17. Wynn, D.C., Eckert C.M., Clarkson, P.J.: Applied signposting: a modeling framework to support design process improvement. In: Proceedings of the ASME International Design Engineering Technical Conferences (2006)

18. Browning, T.R., Eppinger, S.D.: Modeling impacts of process architecture on cost and schedule risk in product development. IEEE Trans. Eng. Manag. 49(4), 428-442 (2002)

19. Krishnan, V., Eppinger, S.D., Whitney, D.E.: A model-based framework to overlap product development activities. Manag. Sci. 43(4), 437-451 (1997)

20. O'Donovan, B.D., Eckert, C.M., Clarkson, P.J.: Simulating design processes to assist in design process planning. In: Proceedings of the ASME Design Engineering Technical Conferences (2004)

21. Lévárdy, V., Browning, T.R.: An adaptive process model to support product development project management. IEEE Trans. Eng. Manag. 56(4), 600-620 (2009)

22. Wyatt, D.F., Wynn, D.C., Jarrett, J.P., Clarkson, P.J.: Supporting product architecture design using computational design synthesis using network structure constraints. Res. Eng. Des. 23(1), 17-52 (2012).

23. Wynn, D.C., Eckert, C.M., Clarkson, P.J.: Modelling iteration in engineering design. In: Proceedings of the 17th International Conference on Engineering Design (2007)

24. Goh, Y.M., McMahon, C.A., Booker, J.: Improving confidence in simulation-based design through error functions. In: Proceedings of the ASME International Design Engineering Technical Conferences (2007)

25. Sébastian, P., Ledoux, Y.: Decision support systems in preliminary design. Int. J. Interact. Des. Manuf. 3(4), 223-226 (2009)

26. Sébastian, P., Chenouard, R., Nadeau, J.-P., Fischer, $\mathrm{X}$.: The embodiment design constraint satisfaction problem of the BOOTSTRAP facing interval analysis and genetic algorithm based decision support tools. Int. J. Interact. Des. Manuf. 1(2), 99-106 (2007)
27. Antonsson, E., Otto, K.N.: Imprecision in engineering design. ASME J. Mech. Des. 117(B), 25-32 (1995)

28. Evans, J.H.: Basic design concepts. J. Am. Soc. Nav. Eng. 71(4), 671-678 (1959)

29. Eppinger, S.D.: Model-based approaches to managing concurrent engineering. J. Eng. Des. 2(4), 283-290 (1991)

30. Jarrett, J.P., Dawes, W.N., Clarkson, P.J.: An approach to integrated multi-disciplinary turbomachinery design. ASME J. Turbomach. 129(3), 488-494 (2007) 31. Jarrett, J.P., Clarkson, P.J.: The surge-stagnate model for complex design. J. Eng. Des. 13(3), 189-196 (2002)

32. Eckert, C.M., Clarkson, P.J., Zanker, W.: Change and customization in complex engineering domains. Res. Eng. Des. 15(1), 1-21 (2004)

33. Bell, C.P., Clarkson, P.J., Dawes,W.N.: Improving the turbine cooling conceptual design process. In: Proceedings of the ASME Turbo Expo 2008, Berlin, Germany, 9-13 May 2008

34. Sobek, D.K. II., Ward, A.C., Liker, J.K.: Toyota's principles of set-based concurrent engineering. Sloan Manag. Rev. 40(2), 67-83 (1999) 